\title{
Clinical and pathological evolution of giant cell arteritis: a prospective study of follow-up temporal artery biopsies in $\mathbf{4 0}$ treated patients
}

\author{
Joseph J Maleszewski ${ }^{1}$, Brian R Younge ${ }^{2}$, John T Fritzlen ${ }^{3}$, Gene G Hunder ${ }^{4}$, Jorg J Goronzy ${ }^{5}$, \\ Kenneth J Warrington ${ }^{4}$ and Cornelia M Weyand ${ }^{5}$ \\ ${ }^{1}$ Department of Laboratory Medicine and Pathology, Mayo Clinic, Rochester, MN, USA; ${ }^{2}$ Department of \\ Ophthalmology, Mayo Clinic, Rochester, MN, USA; ${ }^{3}$ School of Medicine, University of Kansas, Kansas City, \\ KS, USA; ${ }^{4}$ Division of Rheumatology, Mayo Clinic, Rochester, MN, USA and ${ }^{5}$ Division of Immunology and \\ Rheumatology, Stanford University, Stanford, CA, USA
}

\begin{abstract}
Although clinical signs and symptoms of giant cell arteritis improve promptly after starting glucocorticoid therapy, reports have suggested that the vascular inflammation may persist. To assess the duration and quality of histopathologic changes in treated patients, we prospectively obtained second temporal artery biopsies in patients treated for 3 to 12 months after their first diagnostic biopsy. Forty patients (28 women, 12 men, median age 77 years) agreed to have a second temporal artery biopsy randomly assigned to 3, 6, 9, or 12 months subsequent to the first. Clinical and laboratory evaluation of the patient cohort revealed a typical rapid response and continued suppression of clinical manifestations as a result of glucocorticoid treatment. Histopathologic findings, evaluated in a blinded manner by a cardiovascular pathologist, showed unequivocal findings of vasculitis in 7/10 patients with second temporal artery biopsy at 3 months, $9 / 12$ at 6 months, 4/9 at 9 months, and 4/9 at 12 months. Lymphocytes were present in all positive initial biopsies and remained the dominant cell population in chronically treated patients. Granulomatous inflammation decreased in a time-dependent manner from 78 to $100 \%$ at initial biopsy to $50 \%$ at 9 months and $25 \%$ at 12 months. The increased medial fibrosis noted in the second biopsies (60 vs $33 \%$ in primary temporal artery biopsies) suggested that the finding may represent a chronic finding in arteritis. In summary, the response to glucocorticoids in giant cell arteritis was frequently discordant. Clinical manifestations were readily suppressed, but vascular changes were gradual and often incomplete.

Modern Pathology (2017) 30, 788-796; doi:10.1038/modpathol.2017.10; published online 3 March 2017
\end{abstract}

Giant cell arteritis, also referred to as 'temporal arteritis', is the most common vasculitis affecting persons over 50 years old. ${ }^{1,2}$ The disease preferentially affects large- and medium-sized arteries, especially the thoracic aorta and its branches. This tissue tropism explains many of the vascular manifestations of the disease as the involved vessels become narrowed or aneurysmal with possible rupture. Biopsy of a temporal artery, a frequently involved vessel, has been recognized as the gold standard for diagnosis

Investigations over the past two decades suggest that giant cell arteritis is a T-cell-dependent

Correspondence: Dr JJ Maleszewski, MD, Department of Laboratory Medicine and Pathology, Mayo Clinic, 200 First Street SW, Rochester, MN 55905, USA.

E-mail: maleszewski.joseph@mayo.edu

Received 21 November 2016; revised 19 January 2017; accepted 22 January 2017; published online 3 March 2017 inflammatory autoimmune disease with features of an antigen-driven immune response. ${ }^{3-7}$ Granulomatous vascular lesions are populated with T-lymphocytes that secrete INF- $\gamma$ and IL-17 that, in turn, sustain activation of functionally distinct macrophage populations. ${ }^{8}$

Clinical manifestations in giant cell arteritis respond quickly to large doses of glucocorticoids. However, in spite of clinical improvement, biopsies often have shown persistent changes of vasculitis. ${ }^{9-12}$ Such observations suggest some discordance in the systemic and vascular inflammatory components, though systematic studies describing the histopathologic progression of this disease are lacking.

To obtain additional information about the degree and the composition of the vascular inflammation in treated patients, we prospectively obtained second temporal artery biopsies in a cohort of patients whose initial biopsies were positive for giant cell 
arteritis and who received the standard glucocorticoid therapy over a period of a year. Histologic findings were also correlated with clinical symptoms over time as well as outcomes.

\section{Materials and Methods}

\section{Cohort Selection}

Prospective enrollment occurred between 2004 and 2010 of patients with a histopathologic diagnosis of giant cell arteritis made at Mayo Clinic (Rochester, MN, USA). Enrollment required the patient to undergo a second temporal artery biopsy at a randomly assigned interval $(3,6,9$, or 12 months) from the initial diagnosis. Target accrual was 10 patients in each category. This investigation was approved by the Mayo Clinic Institutional Review Board (IRB \#1020-01) and all patients were consented. The nature of the study and the fact that a second biopsy was neither standard practice nor necessary for treatment were fully discussed.

\section{Clinical and Laboratory Methods}

At the first and subsequent visits, medical histories were taken, physical examinations performed, and relevant laboratory tests obtained. Clinical and laboratory findings at the initial and follow-up visits were recorded. Standard clinical laboratory test methods were used.

\section{Biopsy Procedure, Processing, and Histopathologic Examination}

Biopsies were performed under local anesthesia as outpatients by a surgeon (usually BRY), in the Ophthalmology Department. The most clinically suspicious temporal artery was biopsied initially. All biopsies were unilateral. Second biopsies were procured from the contralateral side. In both the biopsies, a 3 to $4 \mathrm{~cm}$ temporal artery specimen was removed and sent for analysis in pathology.

All temporal arteries were handled using standard clinical specimen handling protocols. The specimens were grossly evaluated and serially crosssectioned under the supervision of a surgical pathologist. Eight to 10 complete cross-sections (representing the entire submitted length of vessel) were frozen using a freezing microtome and cut (10 $\mu \mathrm{m}$ thickness), stained with toluidine blue, and intraoperatively evaluated for arteritis. Remaining sections of vessel were formalin-fixed, paraffinembedded, sectioned ( $5 \mu \mathrm{m}$ thickness), stained with hematoxylin and eosin (H\&E) and Verhoeff-van Giesson stains and reviewed by a surgical pathologist. On clinical diagnostic review, all initial cohort specimens showed active giant cell arteritis.

For this study, the biopsy specimens were retrospectively evaluated again in a blinded manner, after enrollment had ended, and documented by a cardiovascular pathologist (JJM) using accepted diagnostic criteria for a diagnosis of arteritis, including the presence of a medial inflammatory infiltrate with mononuclear, or granulomatous features in the temporal artery or a side branch. ${ }^{13}$ The presence or absence of multinucleated giant cells was noted, but not required for the diagnosis. Of note, inflammation restricted to the intima or adventitia was not considered active arteritis. In addition, perivascular inflammation restricted to the vasa vasorum was also not considered active arteritis for the purposes of this study.

The specimens were evaluated histologically for four specific parameters: (i) medial inflammation; (ii) vascular remodeling; (iii) adventitial/peri-vasa vasorum inflammation; (iv) intimal inflammation. Medial inflammation was qualified as primarily lymphocytic, plasmacytic, histiocytic (giant cell), eosinophilic, or neutrophilic. The extent of medial inflammation was graded semi-quantitatively as grade 1 (limited to the immediate region of the internal elastic membrane), grade 2 (beyond the internal elastic membrane, but involving $<50 \%$ of the media), grade 3 (involving $\geq 50 \%$ of the media). The infiltrate itself was characterized also as granulomatous or nongranulomatous. Features of vascular remodeling were also documented, including: medial fibrosis (present or absent); calcification (absent, limited to the internal elastic membrane, or medial involvement); internal elastic membrane disruption/duplication (present or absent); and intimal fibroplasia (absent/mild $(<25 \%$ luminal narrowing) or moderate/severe ( $\geq 25 \%$ luminal narrowing)). Inflammation involving the adventitia or around the small adventitial vessels (peri-vasa vasorum) was also documented and qualified by cell type (lymphocytic, plasmacytic, eosinophilic, neutrophilic, or histiocytic). Inflammation within the intima was noted as either present or absent.

\section{Treatment}

After an initial diagnosis of giant cell arteritis, patients were started on high daily dose prednisone, median dose $60 \mathrm{mg}$ /day (range $30-80 \mathrm{mg} /$ day). The subsequent treatment program was left up to the discretion of the treating physician. In general, 4 weeks after the starting prednisone, the dose was gradually reduced by an average of $10 \%$ of the daily dose every 2 weeks as tolerated. Below 15 to $20 \mathrm{mg}$ per day, the dose was reduced by $2.5 \mathrm{mg}$ decrements every 2 weeks as tolerated and below $10 \mathrm{mg}$ per day, $1 \mathrm{mg}$ decrements were prescribed per month or as tolerated. Patients were followed in person or by correspondence every 1 to 2 months during the study period.

\section{Data Collection and Statistical Analysis}

The study data were collected and managed using REDCap electronic data capture tools hosted at Mayo 
Table 1 Clinical and laboratory findings at initial diagnosis of GCA

Time from initial diagnosis to second $T A B$

\begin{tabular}{|c|c|c|c|c|}
\hline & \\
\hline & 3 months $(\mathrm{n}=10)$ & 6 months $(\mathrm{n}=12)$ & 9 months $(\mathrm{n}=9)$ & 12 months $(\mathrm{n}=9)$ \\
\hline \multicolumn{5}{|l|}{ Clinical finding } \\
\hline Headache & 7 & 10 & 7 & 4 \\
\hline Jaw claudication & 7 & 9 & 6 & 4 \\
\hline Scalp tenderness & 4 & 6 & 3 & 5 \\
\hline Ischemic optic neuropathy & 2 & 1 & 2 & 1 \\
\hline Systemic symptoms & 5 & 5 & 3 & 6 \\
\hline PMR & 3 & 6 & 4 & 2 \\
\hline \multicolumn{5}{|l|}{ Laboratory finding } \\
\hline Hemoglobin $(\mathrm{g} / \mathrm{dl})^{\mathrm{a}}$ & $12.2(10-14.1)$ & $12.9(10.9-13.9)$ & $11.9(10.0-13.4)$ & $10.6(7.2-12.8)$ \\
\hline ESR mm/h ${ }^{a}$ & $57(42-103)$ & $72(16-115)$ & $95(58-130)$ & 74 (53-149) \\
\hline Platelet count $\left(\mathrm{x} 10^{3} / \mu \mathrm{l}\right)^{\mathrm{a}}$ & $408(238-658)$ & $395(228-548)$ & $450(307-598)$ & $420(209-977)$ \\
\hline
\end{tabular}

Abbreviations: ESR, erythrocyte sedimentation rate; PMR, polymyalgia rheumatic; TAB, temporal artery biopsy.

${ }^{\mathrm{a}}$ Median value (range).

Clinic, Rochester, MN, USA. Overall comparisons of the categorical variables were completed using Fishers exact test. The Kruskal-Wallis test was used to compare the laboratory factors among the groups.

\section{Results}

\section{Cohort}

Between 2004 and 2010, 231 patients with clinical findings suggesting giant cell arteritis were seen at the Mayo Clinic for temporal artery biopsy. In 89 $(39 \%)$ of these patients, the biopsy was interpreted to show active arteritis. Forty-one of the 89 patients agreed to participate in this study. On secondary review, by a cardiovascular pathologist, the findings in one patient were considered inadequate for an initial diagnosis of giant cell arteritis and the patient was excluded from further analysis, thus leaving 40 patients in the final study cohort with histopathologically confirmed giant cell arteritis. The final cohort totals were as follows: 3 months $(n=10)$; 6 months $(n=12) ; 9$ months $(n=9) ; 12$ months $(n=9)$.

\section{Clinical and Laboratory Findings}

Twenty-eight of the 40 patients were women and the median age at diagnosis was 77 years (range, 57-89). At the time of the first biopsy, eight patients had taken low doses of prednisone at some time in the past year for the treatment of polymyalgia rheumatic. There was no difference between these and the others regarding symptoms of giant cell arteritis at the time the biopsy was performed (data not shown).

The clinical manifestations related to giant cell arteritis and laboratory tests at admission in the 40 patients categorized according to the time assigned for the second temporal artery biopsy are shown in Table 1. All patients had one or more clinical manifestations of giant cell arteritis. Headaches were present in $28(70 \%)$, jaw claudication in $26(65 \%)$, scalp tenderness in 18 (45\%), polymyalgia rheumatic in $15(38 \%)$, ischemic optic neuropathy in $6(15 \%)$, and systemic symptoms (fatigue, malaise) in 19 $(48 \%)$. There were no significant differences among the four groups regarding the number or type of symptoms $(P>0.05)$.

Laboratory tests were also consistent with giant cell arteritis. The initial hemoglobin concentration was reduced in $29(73 \%)$, erythrocyte sedimentation rate (ESR) elevated in 39 (98\%), and platelet count elevated in $20(50 \%)$. The results were similar among the groups except the median hemoglobin at diagnosis was lower in the 12 -month group $(P=0.01)$. After treatment was started, all of the reversible manifestations improved.

Table 2 lists the clinical findings in the groups at the time of the second temporal artery biopsy. Although all patients with optic neuropathy continued to have some visual deficit, two (one at 9 months, one at 12 months) showed some improved vision at second temporal artery biopsy. Several other patients continued to have mild persistent symptoms. Four of these had mild intermittent headaches, one jaw claudication, one scalp tenderness, one complained of fatigue, and one polymyalgia rheumatic.

Laboratory tests were normal in most at second temporal artery biopsy (Table 2). Values outside the normal range included one patient in the 6-month group with low hemoglobin of $11.7 \mathrm{~g} / \mathrm{dl}$ (positive second temporal artery biopsy), one in the 9-month group with an ESR of $35 \mathrm{~mm} / \mathrm{h}$ (positive second temporal artery biopsy), and one patient in the 12-month group with an ESR of $66 \mathrm{~mm} / \mathrm{h}$ and hemoglobin of $10.6 \mathrm{~g} / \mathrm{dl}$ (negative second temporal artery biopsy). This latter patient also had a small monoclonal protein of undetermined significance, possibly influencing the ESR. All other test results were within normal range at second temporal artery biopsy. 


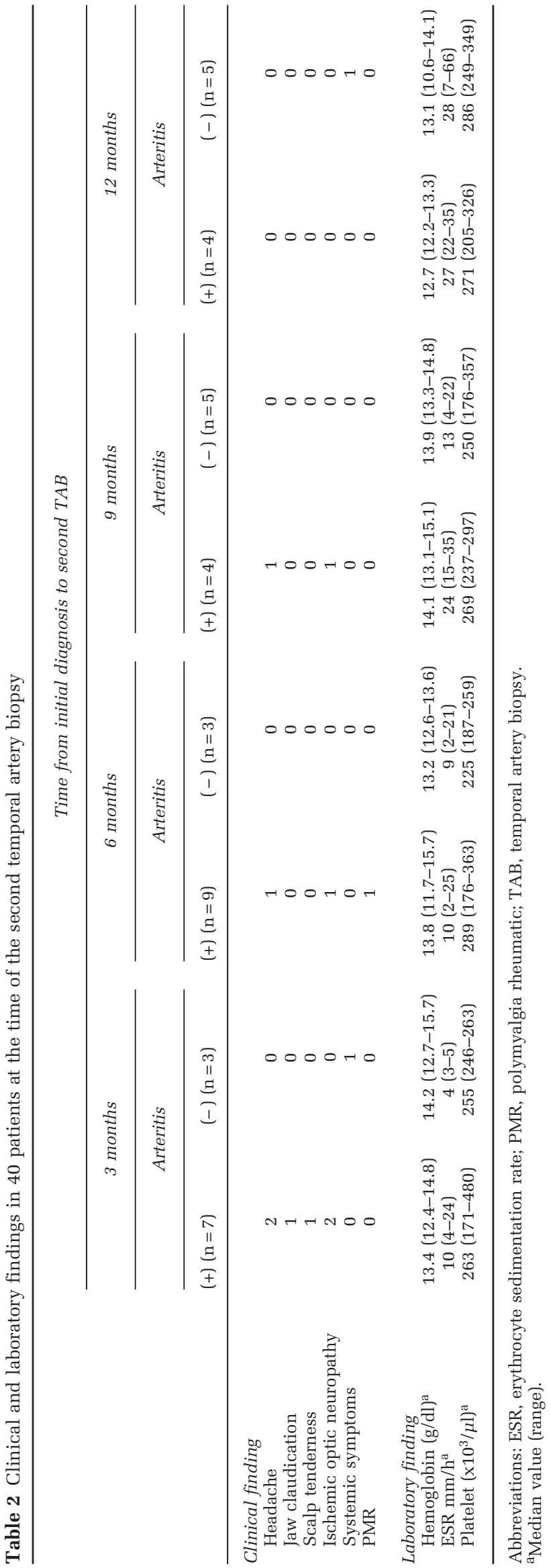

Table 3 shows the results of the second temporal artery biopsies and the dose of prednisone being taken at the time of the second temporal artery biopsy. Patients had been started on reasonably uniform doses, but at second temporal artery biopsy the doses were more varied. The dose was often reduced to near-physiologic levels by 6 months. One asymptomatic patient was no longer taking prednisone at the 12-month follow-up, and had a negative second temporal artery biopsy.

The symptoms that persisted in a minority of patients were not deemed sufficiently indicative of persistent active disease to warrant increasing prednisone dose when weighed against potential adverse effects of glucocorticoid therapy. It is of interest, however, that of the seven patients with persistent symptoms at the time of the second temporal artery biopsy, all except a patient with continued fatigue were in the group with positive second temporal artery biopsies. Thus, the mild continued manifestations might have been clinically important, despite the considerable clinical improvement.

No association was found between the initial manifestations or laboratory results and the results of the second temporal artery biopsy (data not shown). In other words, no specific symptoms or tests predicted the diagnosis at second temporal artery biopsy.

\section{Histopathologic Findings}

The initial temporal artery biopsies showed active giant cell arteritis, consisting of lymphocytic and/or granulomatous inflammation within the arterial media (Figure 1). Lymphocytes were the most common primary cell type encountered. Other cells that were common included plasma cells, 33 (83\%) cases; multinucleated giant cells, 22 (55\%) cases; eosinophils, 13 (33\%) cases; and neutrophils, identified in $1(3 \%)$ case (Table 4). Of note, eosinophils and neutrophils were scant when present. Granulomatous inflammation was observed in 37 (93\%) of the first biopsies.

In the second temporal artery biopsies that had active arteritis, lymphocytes were also the predominant cell type. All four of the other cell types decreased in frequency in the second positive biopsies. Plasma cells were present in less than half on the second positive biopsies. Eosinophils were uncommon initially and rare on the second biopsy. Neutrophils were not seen in any second temporal artery biopsy. Giant cells were identified in $44-67 \%$ of the initial temporal artery biopsies. Of those with active inflammation at second temporal artery biopsy, giant cells were seen in $43 \%$ at 3 months, $78 \%$ at 6 months, and $25 \%$ at 9 months. One case in each of these three cohorts had giant cells at second temporal artery biopsy but had none identified at initial temporal artery biopsy. None of the biopsies examined at 12 months had giant cells. 
Table 3 Biopsy result and prednisone dose at second temporal artery biopsy

Time from initial diagnosis to second $T A B$

\begin{tabular}{lcccc} 
& 3 months $(\mathrm{n}=10)$ & 6 months $(\mathrm{n}=12)$ & 9 months $(\mathrm{n}=9)$ & 12 months $(\mathrm{n}=9)$ \\
\hline Arteritis present $n(\%)$ & $7(70 \%)$ & $9(75 \%)$ & $4(44 \%)$ & $4(44 \%)$ \\
Prednisone dose (mg/day) median (range) & $25(15-50)$ & $9(5-40)$ & $10(2.5-25)$ & $5(0-20)$ \\
\hline
\end{tabular}

Abbreviations: n, number; TAB, temporal artery biopsy.

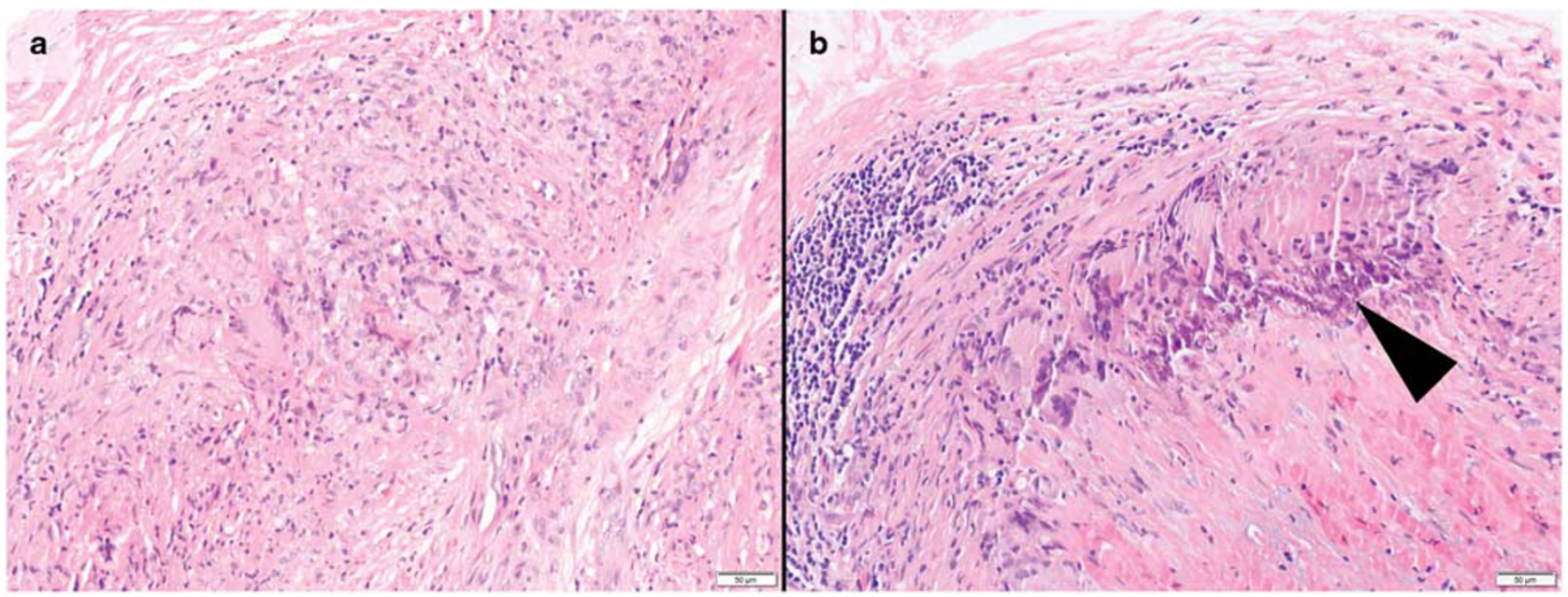

Figure 1 Example Photomicrographs exhibiting (a) a temporal artery with active granulomatous arteritis at the time of initial biopsy and (b) ongoing active granulomatous arteritis at 6 months, post biopsy (hematoxylin and eosin staining; original magnifications, $\times 200$ ). Early calcification is also apparent in the follow-up sample (arrowhead).

Table 4 Character and grade of medial inflammation in biopsies

\begin{tabular}{lcc}
\hline & Initial biopsy & Second biopsy \\
\hline Inflammatory pattern & $(\mathrm{n}=40)$ & $(\mathrm{n}=24)$ \\
$\quad$ Granulomatous & $37(93 \%)$ & $14(58 \%)$ \\
Non-granulomatous & $3(7 \%)$ & $10(42 \%)$ \\
& & \\
Inflammatory cell type & $(\mathrm{n}=40)$ & $(\mathrm{n}=24)$ \\
Lymphocytes & $40(100 \%)$ & $24(100 \%)$ \\
Plasma cells & $33(83 \%)$ & $10(40 \%)$ \\
Giant cells & $22(55 \%)$ & $11(45 \%)$ \\
Eosinophils & $7(18 \%)$ & $1(4 \%)$ \\
Neutrophils & $1(3 \%)$ & $0(0 \%)$ \\
Inflammation grade & & \\
0 & $(\mathrm{n}=40)$ & $(\mathrm{n}=40)$ \\
1 & 0 & 16 \\
2 & 3 & 3 \\
3 & 1 & 4 \\
& 36 & 17 \\
\hline
\end{tabular}

Abbreviations: $n$, number of cases.

${ }^{\mathrm{a}} 0=$ no medial inflammation; $1=$ inflammation limited to the internal elastic membrane; 2 = inflammation beyond internal elastic membrane, but $<50 \%$ of medial thickness; $3=$ inflammation $\geq 50 \%$ of medial thickness.

Granulomatous inflammation was noted in 14 of the 24 positive second temporal artery biopsies. There appeared to be an inverse relationship with granulomatous inflammation and time on therapy,
Table 5 Granulomatous inflammation at different time periods

\begin{tabular}{lcc}
\hline & Initial biopsy n (\%) & Second biopsy n (\%) \\
\hline 3 months & $9(90 \%)$ & $5(71 \%)$ \\
6 months & $12(100 \%)$ & $6(67 \%)$ \\
9 months & $9(100 \%)$ & $2(50 \%)$ \\
12 months & $7(78 \%)$ & $1(25 \%)$ \\
\hline
\end{tabular}

but the numbers are too small to be significant (Table 5 and Figure 2). Adventitial inflammation was present in all of the initial samples and 34 of the follow-up samples (including all 24 cases with positive second temporal artery biopsy; Table 6). In all instances, it was primarily lymphocytic in character, but three initial cases and one follow-up case also had eosinophils present. Inflammation around the small adventitial vessels (peri-vasa vasorum) was noted in 36 initial biopsies and 15 follow-up biopsies (the latter including nine cases of active arteritis). Intimal inflammation was documented in 38 initial temporal artery biopsies and three follow-up temporal artery biopsies.

Positive second temporal artery biopsies were seen in all time periods. Overall, 24 of the $40 \mathrm{~s}$ temporal artery biopsies $(60 \%)$ showed active arteritis. The highest frequency of continued active arteritis on the 


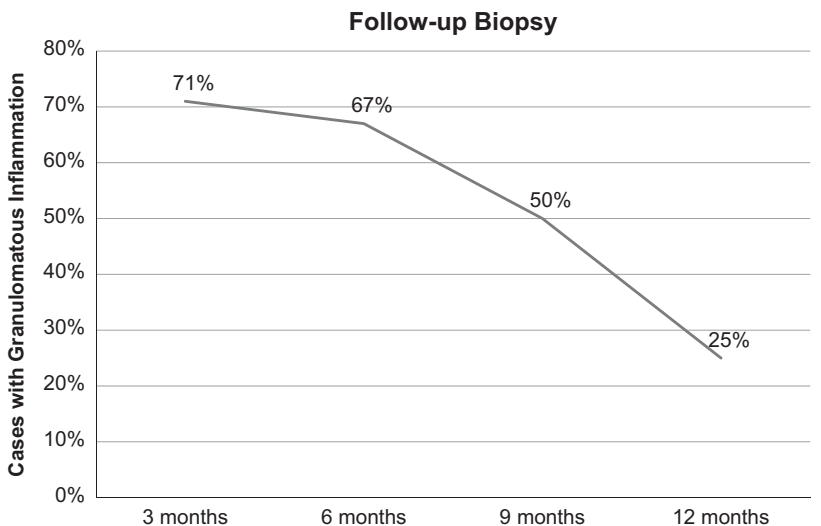

Figure 2 The incidence of granulomatous inflammation in second temporal artery biopsies by cohort. Overall, the incidence of granulomatous inflammation detected in a biopsy is indirectly related to the length of glucocorticoid therapy.

Table 6 Non-medial inflammation in biopsies

\begin{tabular}{lccr}
\hline & & \multicolumn{2}{c}{ Second biopsy } \\
\cline { 3 - 4 } & Initial biopsy & (+) Arteritis & (-) Arteritis \\
\hline Adventitial & $100(100 \%)$ & $24(100 \%)$ & $10(63 \%)$ \\
Peri-vasa vasorum & $30(75 \%)$ & $9(38 \%)$ & $6(38 \%)$ \\
Intimal & $38(95 \%)$ & $1(4 \%)$ & $2(13 \%)$ \\
\hline
\end{tabular}

Table 7 Vascular remodeling

\begin{tabular}{|c|c|c|}
\hline & $\begin{array}{c}\text { Initial biopsy } \\
\mathrm{n}(\%)\end{array}$ & $\begin{array}{c}\text { Second biopsy } \\
\mathrm{n}(\%)\end{array}$ \\
\hline Medial fibrosis & $13(33 \%)$ & $24(60 \%)$ \\
\hline \multicolumn{3}{|l|}{ Calcification } \\
\hline Limited to the IEM & $9(23 \%)$ & $7(18 \%)$ \\
\hline Medial & $1(3 \%)$ & $1(3 \%)$ \\
\hline Disruption/duplication of IEM & $40(100 \%)$ & $39(98 \%)$ \\
\hline \multicolumn{3}{|l|}{ Intimal fibroplasia } \\
\hline Absent $/ \mathrm{mild}^{\mathrm{a}}$ & $37(92 \%)$ & $33(82 \%)$ \\
\hline Moderate/severe ${ }^{\mathrm{b}}$ & $3(8 \%)$ & $7(18 \%)$ \\
\hline
\end{tabular}

Abbreviations: IEM, internal elastic membrane; $n$, number of cases.

${ }^{\mathrm{a} C}$ Causing $<25 \%$ luminal narrowing.

${ }^{\mathrm{b}}$ Causing $\geq 25 \%$ luminal narrowing.

second biopsies was in the first 6 months where 16 of $22(73 \%)$ were positive. But in the 9- and 12-month cohorts, nearly 8 of $18(44 \%)$ biopsies were still positive $(P=0.11)$.

In terms of vascular remodeling, medial fibrosis was noted in 13 of 40 (33\%) of the initial specimens and 24 of the $40(60 \%)$ second biopsies (Table 7 and Figure 3). Calcification was seen in 10 cases at initial biopsy, 9 of which showed only calcification of the internal elastic membrane and 1 with frank medial calcification. Eight follow-up biopsies showed calcification, seven involving only the internal elastic

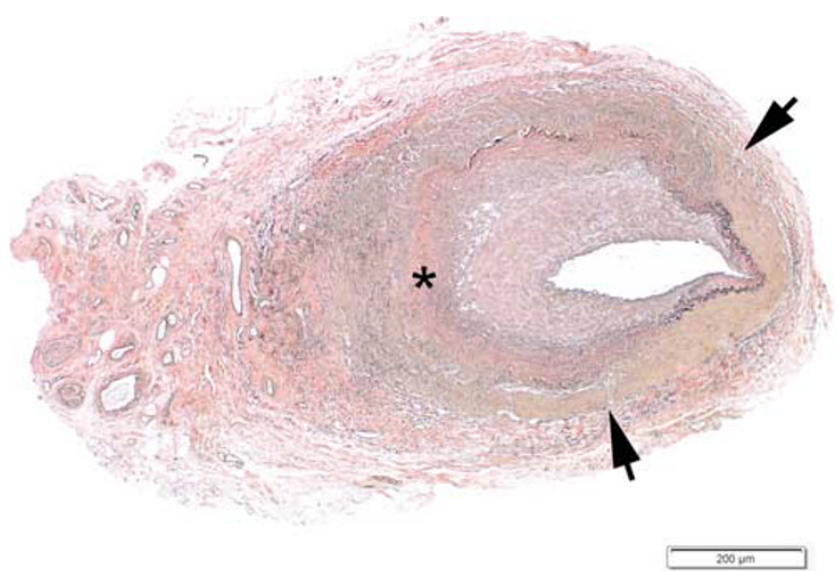

Figure 3 Example photomicrograph showing extensive chronic remodeling, characterized by medial disruption (arrows), extensive medial fibrosis (asterisk) and loss of the internal elastic membrane (Verhoeff-van Giesson staining; original magnification, $\times 40)$.

membrane and one with medial involvement (Figure 3). Disruption or duplication of the internal elastic membrane was seen in all of the initial biopsy specimens, and present in 39 (98\%) of the follow-up samples. Intimal fibroplasia was moderate/severe in $37(93 \%)$ and absent/mild in the balance (Figure 4). On follow-up sampling, moderate/severe intimal fibroplasia was found in $33(83 \%)$ of cases and absent/mild in the balance.

All cases $(n=4)$ with grade 1 or 2 medial inflammation at initial biopsy, were resolved (grade 0) at the second biopsy (Table 4). These included one case in the 6-month cohort, one case in the 9-month cohort, and two cases in the 12-month cohort.

\section{Discussion}

Persistent arteritis in giant cell arteritis after initiation of glucocorticoid therapy has been noted in several retrospective reports. ${ }^{10-12}$ McDonnell et al. ${ }^{9}$ retrospectively evaluated temporal artery biopsies in 237 consecutive patients with varying degrees of elapsed time from initiation of treatment to biopsy and found the longest interval between initiation of therapy and the presence of active arteritis to be 45 days. Those with features of healed arteritis had an average interval of 82 days between starting treatment and temporal artery biopsy. Other scattered reports have shown positive biopsies as long as 6 months after initiation of glucocorticoid therapy. ${ }^{10,14}$ Font and Prabhakaran ${ }^{11}$ noted a single case with active arteritis that had been treated with low-dose corticosteroids for 180 days before a positive biopsy, but noted the inflammation was limited to the media-adventitia junction.

To our knowledge, this investigation is the first prospective study of second temporal artery biopsies that were randomly assigned to time-period cohorts 

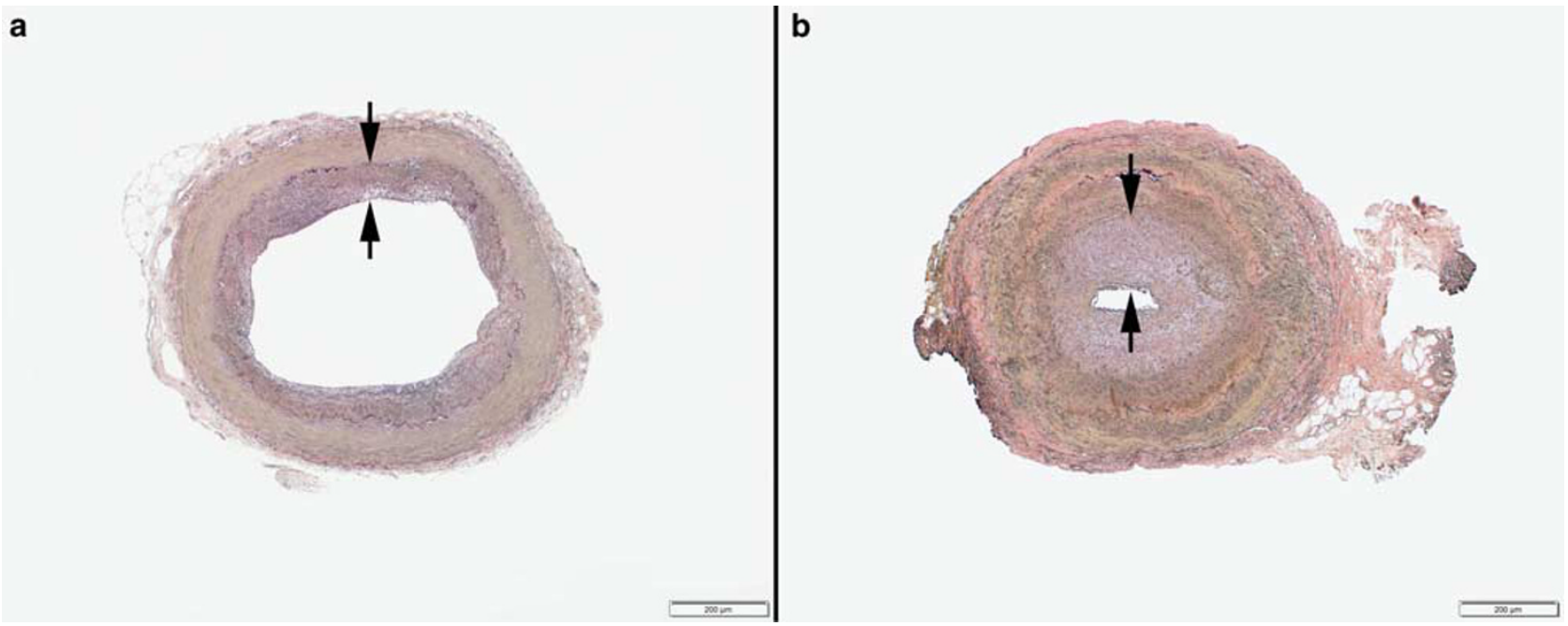

Figure 4 Example photomicrographs showing (a) mild intimal fibroplasia ( $<25 \%$ luminal narrowing, between arrows) and (b) marked intimal fibroplasia ( $>75 \%$ luminal narrowing, between arrows; Verhoeff-van Giesson staining; original magnifications, $\times 40$ ).

over a 1-year period. The patients who participated in the study had clinical and laboratory findings similar to most populations with the disease and experienced rapid improvement of reversible manifestations with glucocorticoid therapy, as is usually experienced. The results of this study showed that in giant cell arteritis the vasculitis may continue for at least one year, despite therapy and extends the longest described interval between glucocorticoid initiation and the presence of histologic arteritis to 420 days.

\section{Clinical Considerations}

Overall, the types and frequencies of symptoms were typical of giant cell arteritis, at initial diagnosis. The variability of clinical findings among the four groups was not major and was unlikely a critical determinant of the results. However, a minority of patients $(20 \%)$ had continued mild clinical manifestations at second temporal artery biopsy that were less marked than at initial presentation, but which could be, in retrospect, related to active disease. The majority of these were in the group who had temporal artery biopsies 3 months after diagnosis and mainly in the group with persistently positive biopsies and therefore could be attributed to ongoing arteritis. However, the numbers were too small to meaningfully analyze. These milder continuing clinical manifestations were not considered by the clinicians caring for the patients as clear evidence of persistent arteritis and the prednisone reduction schedule was continued or slowed. None of the clinical parameters at the time of diagnosis were found to help predict the results of the second temporal artery biopsy.

No patients in the study developed a significant complication of giant cell arteritis after initiation of therapy. The ischemic ocular findings at diagnosis persisted; however, two patients with optic neuropathy had partial improvement of vision by second biopsy. Data regarding long-term vascular outcomes for these patients (eg, aneurysm formation) are not currently available. Clinical relapses or recurrences have been recorded in some cases of giant cell arteritis. ${ }^{15}$ We are uncertain if they had a role in our findings, but none were observed by us with careful follow-up.

The median dose of prednisone prescribed was progressively lower over the study period but varied for each patient. One of the patients with a negative second temporal artery biopsy at 9 months was in clinical remission and on only $2.5 \mathrm{mg}$ of prednisone per day and one in the 12-month group was off prednisone completely without symptoms or abnormal tests and a negative second temporal artery biopsy.

The results of this study indicate that a patient with giant cell arteritis is a complex picture and suggests that biopsy findings alone are not an absolute indication for high-dose glucocorticoids. Rather, the history of the course of clinical manifestation and treatment must be brought to bear in clinical decision-making. The findings also lend support to debunking the notion that treatment with glucocorticoids for several days or even weeks will result in a false-negative biopsy, and underscore the utility of temporal artery biopsy even in treated patients. Persistence of histopathologic changes despite treatment suggests that this disease evolves over a longer period than previously thought.

\section{Histopathologic Considerations}

For the pathologist evaluating samples from patients already treated for giant cell arteritis, this study describes important changes over time in a treated cohort. Granulomatous features, for example, while near universal early in the course of the disease, tend 
to diminish with treatment and the absence of such features should not necessarily be taken as indicative of an alternative form of vasculitis.

The overall frequency of histologic vasculitis on the second biopsy was higher than expected at $60 \%$. If the first two periods ( 3 and 6 months) and last two periods (9 and 12 months) are grouped together, the decline in combined frequencies from $73 \%$ to $44 \%$ suggests a (slowly) subsiding inflammatory reaction. We cannot be certain whether the regression of the inflammation would continue over additional months.

The morphology of the inflammation at both the initial and second biopsies was similar and in keeping with the histopathologic spectrum of giant cell arteritis. The character of the inflammation was granulomatous in nearly all cases initially (93\%), but fell in a time-dependent manner such that it was in only $25 \%$ of positive cases at 12 months.

There were also changes in the inflammatory constituency noted between the first and second biopsies. Aside from lymphocytes, which were the predominant cell type in both initial and second biopsies, changes were observed in the frequency of the other cell types. The frequency of giant cells went from approximately half in the initial specimens to about one-fourth on the second biopsy and fell in a time-dependent manner. The clearance of giant cells early in the course of immunosuppressive therapy has been described in other inflammatory conditions, such as giant cell myocarditis. ${ }^{16}$

Non-medial inflammation, involving the intima or peri-vasa vasorum was very common at the time of initial diagnosis, but much less so following glucocorticoid therapy. Adventitial inflammation, on the other hand was almost universally encountered in both initial and follow-up samples.

Most changes indicative of vascular remodeling were seen at similar frequency in the initial and follow-up biopsies. Medial fibrosis was more common in the follow-up biopsies (60 vs 33\%), likely a reflection of the chronic healing process. Calcification did not appear to be an indicator of chronicity in this series.

\section{Study Limitations}

The study is limited primarily by its size. Although it is the largest of its kind, certain trends related to the histopathologic progression did not achieve statistical significance. In addition, given the patchy, but multifocal nature of giant cell arteritis, sampling is often an issue - particularly within a limited population. Nevertheless, this latter issue is true also with respect to clinical practice.

\section{Conclusions and Future Directions}

The current study supports the emerging concept that two relatively independent disease components contribute to giant cell arteritis. ${ }^{15}$ One component, reflected in the clinical signs and symptoms of systemic inflammation, is molecularly defined by an intense acute phase response, likely moderated by the liver and lymphoid organs. ${ }^{17}$ The second component, which is captured in the current study, is the vascular inflammation. Why the two components are, at least partially, uncoupled is not understood. Molecular analysis of the vascular lesions has demonstrated that three cellular drivers build stable granulomatous infiltrates: CD4 ${ }^{+}$T-cells, highly activated macrophages, and dendritic cells. ${ }^{18}$ Although antigen has been suspected to function as a trigger of intramural inflammation, more recent data point towards defects in the threshold setting of the immune system, leading to unopposed T-cell activation in the vessel wall.6,7,19

Deficiencies in immune checkpoint regulation, particularly the PD-1 immune checkpoint, have been implicated in compromising the immuno-privilege of the arterial wall. ${ }^{20}$ Also, giant cell arteritis patients lack a population of immune-inhibitory $\mathrm{CD}^{+}$ T-regulatory cells, which fosters unopposed expansion and activation of polyclonal CD4 ${ }^{+}$T-cells. $^{21}$ Molecular analysis of the vasculitic lesions has demonstrated that multiple functional lineages contribute to the granulomatous infiltrates and that such functionally lineages are differentially susceptible to therapeutic interventions. ${ }^{22}$ Specifically, IL-17producing $\mathrm{T}$-cells disappear from the lesions in steroid-treated patients, while IFN- $\gamma$-producing T-cells persist. ${ }^{8,23}$ The multifunctionality of vesselwall residing $\mathrm{T}$-cells questions the role of a single antigen in driving the misplaced immune response. The current study supports the notion that T-cells are particularly persistent. Dwelling times of vasculitogenic T-cells in the wall infiltrates are currently unknown, but data presented here would suggest that lesional T-cells can reside in the arterial wall for years.

In the current study, tissue-residing macrophages appeared more responsive to corticosteroidmediated immune suppression than T-cells. This finding is supportive of the model that macrophages follow, and not precede, T-cells into the vessel wall. $^{24}$ Profiling of tissue cytokines in temporal artery specimens from untreated and treated patients have indicated differential responsiveness of monokines, in line with the concept that several macrophage subpopulations participate in the granulomatous infiltrates. ${ }^{8}$ Functional assignment of macrophages in temporal artery lesions have revealed a tight association between positioning of macrophages and their functional profile. ${ }^{25,26}$ More detailed molecular studies are required to better understand which subset of macrophages fulfill the different pathogenic functions in the vasculitic lesions. The gatekeeper function of recruiting inflammatory cells, first T-cells, then macrophages into the immuno-privileged niche of the arterial wall appears to lie with vascular dendritic cells. ${ }^{27}$ Their fate over 
the course of giant cell arteritis requires further investigation.

In essence, giant cell arteritis transitions from acute into chronic vasculitis, even in patients treated with high-dosed corticosteroids. Vascular infiltrates are remarkably stable, particularly vessel-wall infiltrating T-cells. Although the systemic component of giant cell arteritis is swiftly responsive to corticosteroids, vessel wall infiltrates persist over months to years, emphasizing the local tissue environment as a critical pathogenic factor.

\section{Disclosure/conflict of interest}

The authors declare no conflict of interest.

\section{References}

1 Aiello PD, Trautmann JC, McPhee TJ, et al. Visual prognosis in giant cell arteritis. Ophthalmology 1993; 100:550-555.

2 Evans JM, Batts KP, Hunder GG. Persistent giant cell arteritis despite corticosteroid treatment. Mayo Clin Proc 1994;69:1060-1061.

3 Weyand CM, Goronzy JJ. Giant cell arteritis as an antigen-driven disease. Rheum Dis Clin North Am 1995;21:1027-1039.

4 Brack A, Geisler A, Martinez-Taboada VM, et al. Giant cell vasculitis is a T cell-dependent disease. Mol Med 1997;3:530-543.

5 Weyand CM, Younge BR, Goronzy JJ. T cells in arteritis and atherosclerosis. Curr Opin Lipidol 2008;19:469-477.

6 Weyand CM, Liao YJ, Goronzy JJ. The immunopathology of giant cell arteritis: diagnostic and therapeutic implications. J Neuroophthalmol 2012;32:259-265.

7 Hilhorst M, Shirai T, Berry G, et al. T cell-macrophage interactions and granuloma formation in vasculitis. Front Immunol 2014;5:432.

8 Deng J, Younge BR, Olshen RA, et al. Th17 and Th1 T-cell responses in giant cell arteritis. Circulation 2010;121:906-915.

9 McDonnell PJ, Moore GW, Miller NR, et al. Temporal arteritis. A clinicopathologic study. Ophthalmology 1986;93:518-530.

10 Ray-Chaudhuri N, Kine DA, Tijani SO, et al. Effect of prior steroid treatment on temporal artery biopsy findings in giant cell arteritis. Br J Ophthalmol 2002;86: 530-532.

11 Font RL, Prabhakaran VC. Histological parameters helpful in recognising steroid-treated temporal arteritis: an analysis of 35 cases. Br J Ophthalmol 2007;91:204-209.
12 Achkar AA, Lie JT, Hunder GG, et al. How does previous corticosteroid treatment affect the biopsy findings in giant cell (temporal) arteritis? Ann Intern Med 1994;120:987-992.

13 Miller DV, Maleszewski JJ. The pathology of largevessel vasculitides. Clin Exp Rheumatol 2011;29: S92-S98.

14 Guevara RA, Newman NJ, Grossniklaus HE. Positive temporal artery biopsy 6 months after prednisone treatment. Arch Ophthalmol 1998;116:1252-1253.

15 Weyand CM, Goronzy JJ. Clinical practice. Giant-cell arteritis and polymyalgia rheumatica. N Engl J Med 2014;371:50-57.

16 Maleszewski JJ, Orellana VM, Hodge DO, et al. Longterm risk of recurrence, morbidity and mortality in giant cell myocarditis. Am J Cardiol 2015;115: 1733-1738.

17 Weyand CM, Goronzy JJ. Immune mechanisms in medium and large-vessel vasculitis. Nat Rev Rheumatol 2013;9:731-740.

18 Wagner AD, Goronzy JJ, Weyand CM. Functional profile of tissue-infiltrating and circulating CD68+ cells in giant cell arteritis. Evidence for two components of the disease. J Clin Invest 1994;94:1134-1140.

19 Ma-Krupa W, Jeon MS, Spoerl S, et al. Activation of arterial wall dendritic cells and breakdown of selftolerance in giant cell arteritis. J Exp Med 2004;199: 173-183.

20 Zhanga $\mathrm{H}$, Watanabea R, Berry $\mathrm{G}$, et al. Immunoinhibitory checkpoint deficiency in medium and large vessel vasculitis. Proc Natl Acad Sci USA 2017;114: E970-E979.

21 Wen Z, Shimojima Y, Shirai T, et al. NADPH oxidase deficiency underlies dysfunction of aged CD8+ Tregs. J Clin Invest 2016;126:1953-1967.

22 Watanabe R, Hosgur E, Zhang H, et al. Pro-inflammatory and anti-inflammatory $\mathrm{T}$ cells in giant cell arteritis. Joint Bone Spine 2016;S1297-319X:30124-30125.

23 Weyand CM, Younge BR, Goronzy JJ. IFN- $\gamma$ and IL-17: the two faces of T-cell pathology in giant cell arteritis. Curr Opin Rheumatol 2011;23:43-49.

24 Shirai T, Hilhorst M, Harrison DG, et al. Macrophages in vascular inflammation-from atherosclerosis to vasculitis. Autoimmunity 2015;48:139-151.

25 Weyand CM, Wagner AD, Bjornsson J, et al. Correlation of the topographical arrangement and the functional pattern of tissue-infiltrating macrophages in giant cell arteritis. J Clin Invest 1996;98:1642-1649.

26 Rittner HL, Kaiser M, Brack A, et al. Tissue-destructive macrophages in giant cell arteritis. Circ Res 1999;84: 1050-1058.

27 Pryshchep O, Ma-Krupa W, Younge BR, et al. Vesselspecific Toll-like receptor profiles in human medium and large arteries. Circulation 2008;118:1276-1284. 